Louisiana State University

LSU Digital Commons

Faculty Publications

Department of Physics \& Astronomy

$12-7-2007$

\title{
Attosecond control of ionization by wave-packet interference
}

P. Johnsson

Lunds Universitet

J. Mauritsson

Lunds Universitet

T. Remetter

Lunds Universitet

A. L'Huillier

Lunds Universitet

K. J. Schafer

Louisiana State University

Follow this and additional works at: https://digitalcommons.Isu.edu/physics_astronomy_pubs

\section{Recommended Citation}

Johnsson, P., Mauritsson, J., Remetter, T., L'Huillier, A., \& Schafer, K. (2007). Attosecond control of ionization by wave-packet interference. Physical Review Letters, 99 (23) https://doi.org/10.1103/

PhysRevLett.99.233001

This Article is brought to you for free and open access by the Department of Physics \& Astronomy at LSU Digital Commons. It has been accepted for inclusion in Faculty Publications by an authorized administrator of LSU Digital Commons. For more information, please contact ir@lsu.edu. 


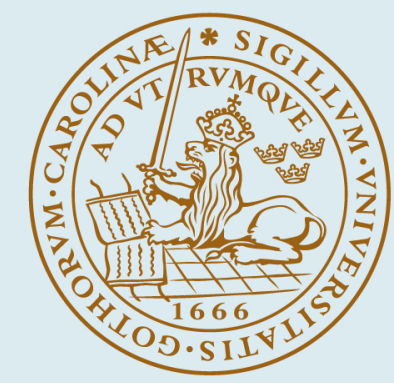

\section{LUND UNIVERSITY}

\section{Attosecond control of ionization by wave-packet interference}

Johnsson, Per; Mauritsson, Johan; Remetter, Thomas; L'Huillier, Anne; Schafer, K J

Published in:

Physical Review Letters

DOI:

10.1103/PhysRevLett.99.233001

2007

Link to publication

Citation for published version (APA):

Johnsson, P., Mauritsson, J., Remetter, T., L'Huillier, A., \& Schafer, K. J. (2007). Attosecond control of ionization by wave-packet interference. Physical Review Letters, 99(23). https://doi.org/10.1103/PhysRevLett.99.233001

Total number of authors:

5

\section{General rights}

Unless other specific re-use rights are stated the following general rights apply:

Copyright and moral rights for the publications made accessible in the public portal are retained by the authors

and/or other copyright owners and it is a condition of accessing publications that users recognise and abide by the

legal requirements associated with these rights.

- Users may download and print one copy of any publication from the public portal for the purpose of private study

or research.

- You may not further distribute the material or use it for any profit-making activity or commercial gain

- You may freely distribute the URL identifying the publication in the public portal

Read more about Creative commons licenses: https://creativecommons.org/licenses/

Take down policy

If you believe that this document breaches copyright please contact us providing details, and we will remove

access to the work immediately and investigate your claim. 


\title{
Attosecond Control of Ionization by Wave-Packet Interference
}

\author{
P. Johnsson, ${ }^{1, *}$ J. Mauritsson, ${ }^{1,2}$ T. Remetter, ${ }^{1}$ A. L'Huillier, ${ }^{1}$ and K. J. Schafer ${ }^{2}$ \\ ${ }^{1}$ Department of Physics, Lund University, P. O. Box 118, SE-221 00 Lund, Sweden \\ ${ }^{2}$ Department of Physics and Astronomy, Louisiana State University, Baton Rouge, Louisiana 70803-4001, USA \\ (Received 24 August 2007; published 7 December 2007)
}

A train of attosecond pulses, synchronized to an infrared (IR) laser field, is used to create a series of electron wave packets (EWPs) that are below the ionization threshold in helium. The ionization probability is found to strongly oscillate with the delay between the IR and attosecond fields twice per IR laser cycle. Calculations that reproduce the experimental results demonstrate that this ionization control results from interference between transiently bound EWPs created by different pulses in the train. In this way, we are able to observe, for the first time, attosecond wave-packet interference in a strongly driven atomic system.

DOI: 10.1103/PhysRevLett.99.233001

PACS numbers: $32.80 . \mathrm{Rm}, 32.80 . \mathrm{Qk}, 42.65 . \mathrm{Ky}$

Attosecond pulses [1,2] can be used to initiate and control electron dynamics on a sub-femtosecond time scale [3]. The first step in this process occurs when an atom absorbs an ultraviolet photon leading to the formation of an attosecond electron wave packet (EWP). Until now, attosecond pulses have been used to create EWPs in the continuum above the first ionization threshold, where they quickly disperse [4-8]. When synchronized to an infrared (IR) laser field, these pulses can be used to control the time at which ionization occurs, but not its probability. In this Letter we demonstrate that using attosecond pulses tuned to create EWPs below the ionization threshold allows for the control of both the timing and the probability of ionization on an attosecond time scale.

In our attosecond wave-packet experiments we use a train of ultraviolet (UV) attosecond pulses to ionize either helium or argon atoms in the presence of an IR field. The attosecond pulses are phase locked to the IR field since their spacing in time is precisely one half of the laser period. The central energy of the pulses, $\approx 23 \mathrm{eV}$, is higher than the ionization energy of argon $(15.8 \mathrm{eV})$, but below that of helium $(24.6 \mathrm{eV})$, as shown in Fig. 1(a). We demonstrate the ability to control the ion yield in helium through the delay between the two fields, an effect which is absent in argon. We attribute the ionization control in helium to interference between transiently bound EWPs which can modulate the probability that an electron is excited out of the atomic ground state. Calculations based on integration of the time-dependent Schrödinger equation (TDSE) show that the contrast in the ionization probability versus the IR-UV delay is an order of magnitude larger than what is achieved with a single pulse, and that the contrast grows as the number of pulses in the train is increased.

The modulation of photon absorption by wave-packet interference (WPI) has been used in molecules as a probe of nuclear dynamics on the femtosecond time scale [9-11], and in Rydberg atoms as a probe of electron dynamics on a picosecond time scale $[12,13]$. It is a sensitive tool for probing quantum dynamics because it depends on the spatial and temporal behavior of the wave packets in the confining potential. The modulation in the total amount of population excited out of the ground state results from the interference between absorption and stimulated emission, and can only occur if some part of an initially localized wave packet returns to the region of space where it was created during the time when a subsequent wave packet is excited. In this Letter we show for the first time evidence for this effect in the attosecond domain and for a strongly driven system.

The attosecond pulse train (APT) used to excite the atoms was synthesized from high-order harmonics generated in xenon by focusing $35 \mathrm{fs}, 796 \mathrm{~nm}(1.56 \mathrm{eV}$ photon energy) pulses from a $1 \mathrm{kHz}$ Ti:sapphire laser to an intensity of $\approx 8 \times 10^{13} \mathrm{~W} \cdot \mathrm{cm}^{-2}$ in a $3 \mathrm{~mm}$ long windowless gas cell filled to a static pressure of $\approx 20 \mathrm{mbar}$. The APT was filtered spatially by passing it through a $1.5 \mathrm{~mm}$ diameter aperture, and spectrally using a $200 \mathrm{~nm}$ thick
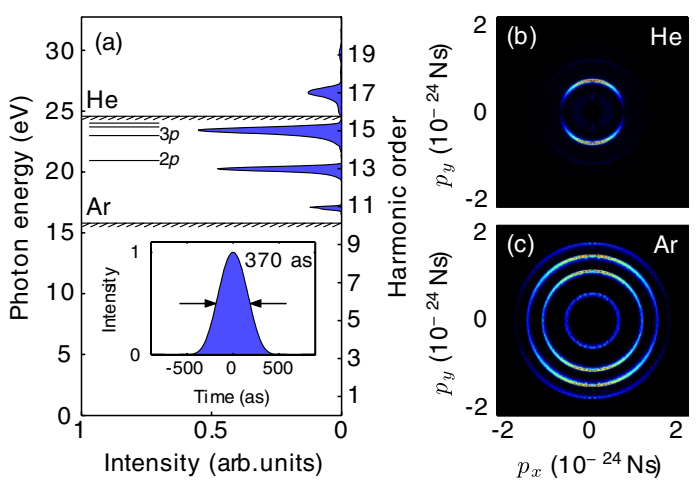

FIG. 1 (color online). (a) Spectrum of the UV pulses used in the experiment shown in relation to the ionization potentials of helium and argon. The inset shows the temporal profile of the attosecond pulses in the train. Experimental photoelectron momentum distributions from single-photon ionization by the APT in helium (b) and argon (c) with the polarization of the light parallel to the $p_{y}$ axis. 
aluminum filter. The spatial filter removes contributions to the harmonic emission from the longer quantum paths, while the aluminum filter blocks the remaining IR and the intense low-order harmonics [14]. The spectrum of the APT is shown in Fig. 1(a) and consists of harmonics 11 to 17 , with a central energy of $23 \mathrm{eV}$. The pulses were characterized using the RABITT technique (reconstruction of attosecond beating by interference of two-photon transitions) [1,15], and the average duration of the bursts was found to be 370 as with the temporal profile shown in the inset in Fig. 1(a).

A velocity map imaging spectrometer was used for detection, having the advantage of being able to operate either in electron imaging [16] or in ion time-of-flight mode. For both ion and electron detection, the target gas was injected by means of an atomic beam pulsed at $50 \mathrm{~Hz}$. The $2 \mathrm{D}$ projections of the momentum distributions of the photoelectrons were recorded by means of an MCPassembly and a CCD-camera, and the 3D momentum distributions were obtained using the iterative inversion procedure described in [17]. Examples of momentum distributions obtained from UV ionization are shown in Figs. 1(b) and 1(c) for helium and argon, respectively.

The dressing IR pulse, a delayed replica of the pulse generating the harmonics, was collinearly overlapped with the APT before both beams were refocused into the spectrometer by a toroidal platinum mirror. The IR intensity was determined to be $1.3 \times 10^{13} \mathrm{~W} \cdot \mathrm{cm}^{-2}$, by using ion yields in xenon as a function of intensity as well as the ponderomotive shift in the photoelectron spectra [18] to accurately calibrate the intensity. The APT envelope being substantially shorter than that of the IR pulse (10 fs vs $35 \mathrm{fs}$ ) and the UV focal spot being smaller than that of the IR, means that all of the atoms in the interaction volume excited by UV absorption were exposed to approximately the same IR intensity. A crucial point is that this laser intensity is too low to excite any population out of the argon or helium ground states by itself. This means that the ground state is connected to the excited bound and continuum states only when an attosecond pulse is present, an essential condition for observing WPI. Also of importance is the fact that although the IR laser field is weak from the point of view of an electron in the ground state, it is strong from the point of view of an electron excited out of the ground state. At peak amplitude, the IR field suppresses the Coulomb potential by $\sim 7 \mathrm{eV}$ at the saddle point, which is enough to unbind all of the single excited bound states of helium. Furthermore, as the barrier suppression changes very slowly with intensity, scaling as $I^{1 / 4}$, our method results in creating free attosecond EWPs in a strong oscillating laser field. The absolute timing between the APT and the IR field was not accessible experimentally, and has been chosen to fit the results of the calculations.

Figure 2 shows our main experimental result, the delay dependence of the ion yields, $P_{\text {ion }}$, from helium and argon.

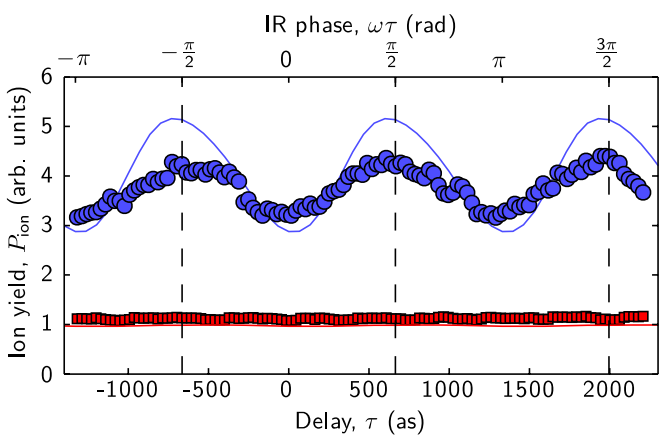

FIG. 2 (color online). Experimentally measured ion yields, $P_{\text {ion }}$, for $\mathrm{He}^{+}$(blue circles) and $\mathrm{Ar}^{+}$(red squares) as a function of the delay $\tau$ between the attosecond pulses and the IR field. Calculated ion yields in the same conditions $\left(\mathrm{He}^{+}\right.$, blue solid line; $\mathrm{Ar}^{+}$red solid line).

We use a sine convention for the IR electric field so that delays, $\tau$, which are multiples of $\pi / \omega=1330$ as, where $\omega$ is the IR laser frequency ( $\hbar \omega=1.56 \mathrm{eV})$, correspond to the attosecond pulses overlapping the zero-crossings of the IR field. All yields are normalized to those obtained with only the APT present. For $\mathrm{Ar}^{+}$(red squares), there is no measurable effect of the IR field while for $\mathrm{He}^{+}$(blue circles) the ion yield is increased by a factor of 4 when the IR field is present. In addition, the $\mathrm{He}^{+}$yield exhibits a modulation as a function of the UV-IR delay. The depth of the modulation is $\approx 35 \%$ and the period is equal to half the laser period. Modulations were also observed at other IR intensities, with similar or slightly reduced contrast.

To gain insight into the results presented in Fig. 2, we have performed calculations based on the integration of the TDSE $[19,20]$ and using the single active electron approximation. The atomic potentials used were the standard Hartree-Fock potential for helium, and a pseudopotential in argon [21], which reproduce the single electron excited states very well. To simulate the experiments we use an IR pulse whose electric field envelope is a cosine function with a FWHM in intensity of $35 \mathrm{fs}$, and an APT whose electric field envelope is a somewhat sharper cosine square function with a FWHM in intensity of $10 \mathrm{fs}$. The total population excited was calculated as one minus the population remaining in the ground state at the end of the pulse, while the total ionization was calculated either from the photoelectron spectrum [22] or by running the calculation for 10 additional IR cycles and calculating the probability to remain in the vicinity of the ion.

The ion yields obtained at the end of the interaction are indicated in Fig. 2 as solid red and solid blue lines for argon and helium, respectively, showing good agreement with the experiment. The calculations also show that without an IR field, the ionization probability in $\mathrm{He}$ is equal to the absorption probability, meaning that no population is left in the excited bound states. As indicated in Fig. 1(a), the spectrum of the UV pulses overlaps poorly with the acces- 
sible excited bound states of helium, and the atom is limited to absorbing photons belonging to the 17th harmonic, leading to immediate ionization.

In Fig. 3(a), we show more complete theoretical results for He. Shown are both the photo absorption probability that an electron is excited out of the ground state $\left(P_{\mathrm{abs}}\right.$, blue line) and the ionization probability ( $P_{\text {ion }}$, red line). The difference between these probabilities is the probability to remain trapped in an excited state after the IR pulse ends $\left(P_{\text {trp }}\right.$, green line). Two features are immediately apparent. First, the modulation in the $\mathrm{He}^{+}$yield is caused by the fact that the amount of population excited out of the ground state by the APT in the presence of the IR field is modulated as a function of UV-IR delay. Second, the ionization of the population promoted out of the ground state by the APT is incomplete, leaving $30 \%-40 \%$ of the promoted population in excited states after the IR field is over.

The delay dependence of the $\mathrm{He}^{+}$yield has two contributions. First, each pulse in the APT excites population in the presence of an IR field that distorts the atomic potential by an amount that depends on the IR-UV delay. A single attosecond pulse would therefore probe solely the atom's ability to absorb light near the ionization threshold in the presence of an electric field which can be as high as

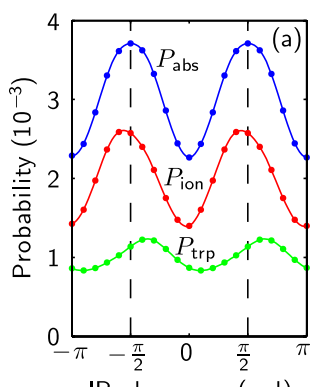

IR phase, $\omega \tau$ (rad)

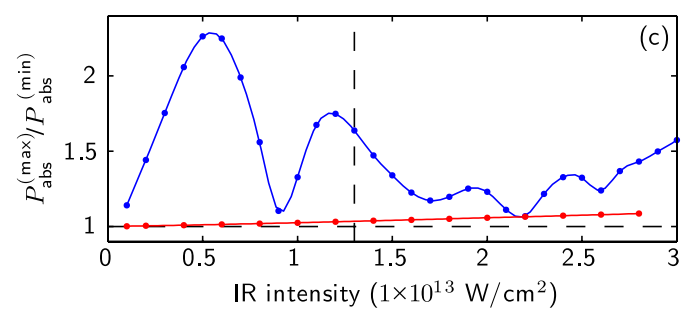

FIG. 3 (color online). (a) Calculated probabilities for removal of an electron from the ground state $\left(P_{\mathrm{abs}}\right.$, blue line), ionization $\left(P_{\text {ion }}\right.$, red line) and remaining in an excited bound state $\left(P_{\text {trp }}\right.$, green line) as a function of the phase of the IR field at the time of the attosecond pulses. (b) Absorption probability, $P_{\text {abs }}$, versus delay for different APTs, normalized to the excitation probability for zero delay in each case. The FWHM of the APT intensity envelope is $1 \mathrm{fs}$ (single attosecond pulse, blue line), 2 fs (red line), 4 fs (green line) or $8 \mathrm{fs}$ (black line). (c) Contrast (defined as the maximum excitation probability divided by the minimum) for various peak intensities of the IR field for a single pulse (red line) and for a train with $10 \mathrm{fs}$ FWHM (blue line). The dashed vertical line indicates the experimental intensity. $\sim 10^{8} \mathrm{~V} / \mathrm{cm}$. Our calculations show that the modulation in the ion yield due to such a single attosecond pulse is about 1\%-3\% over the intensity range covered by the experiment, 10 times smaller than the observed effect. The other contribution to the delay dependence is from WPI. This temporal interference in the total excitation probability comes about if an EWP created by one pulse in the train has some probability to be near the ion core when a later packet is being excited by a different pulse in the train. This requires that an EWP excited by a single pulse takes more that one half cycle to completely ionize. Indeed, at all delays we find that the EWP excited by a single attosecond pulse takes one to several IR cycles to completely ionize, fulfilling this condition for WPI.

WPI also causes the excitation probability to scale nonlinearly with the number of pulses in the train. In the absence of WPI the relative modulation in the total excitation probability versus delay is the same for different length pulse trains. In Fig. 3(b), we plot the normalized excitation probability for APTs of different length, changing from a single pulse (the $1 \mathrm{fs}$ envelope) to two or more. We see that the relative modulation increases as the APT length is increased. We also note that the delay curve reverses its shape when the number of pulses is increased from one to two or more. In argon, by contrast, the total excitation is linear in the length of the pulse train.

By its nature, WPI is a very sensitive probe of the electron dynamics in a bound system. In our system these dynamics are most easily altered by changing the IR intensity. In Fig. 3(c) we plot the magnitude of the calculated relative modulation (i.e. the contrast) versus peak IR intensity for intensities ranging from 0.1 to $3.0 \times 10^{13} \mathrm{~W}$. $\mathrm{cm}^{-2}$ and a $10 \mathrm{fs}$ APT (blue line). As can be seen, the contrast is a very sensitive function of the field amplitude. For comparison, the contrast from using a single 370 as pulse is shown (red line). In this same range of intensities the amount of population ionized after the IR pulse is over ranges from $40 \%-100 \%$ of the total population excited out of the ground state, and exhibits a very complicated dependence on the IR intensity.

Additional support for the WPI picture that we present can be found in the experimental measurements of the energy-resolved angular distributions from helium and argon, presented in Fig. 4. The momentum distributions from argon [Figs. 4(a) and 4(b)] show the difference between the two delays that results in the greatest and least number of high energy electrons. The highest energy electrons [Fig. 4(b)] are created when the attosecond pulses are timed so that ionization takes place at the zero crossings of the electric field $(\omega \tau=n \pi)$, when the momentum transfer from the field to the electronic wave packet is maximum [6,7]. In this case the IR field only redistributes the energy of the ionized electrons, depending on the phase of the IR field at the time they enter the continuum, and the angular distributions remain rather broad for all delays. In contrast 

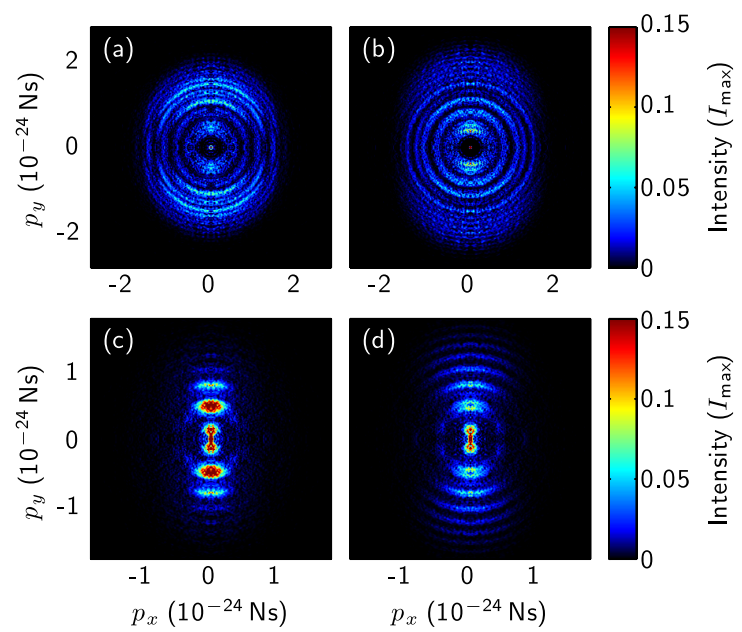

FIG. 4 (color online). Momentum distributions from argon [(a) and (b)] and helium [(c) and (d)] for the IR-UV delays corresponding to lowest [(a) and (c)] and highest [(b) and (d)] maximum electron momentum along the $p_{y}$ axis. The polarization directions of the UV and IR fields are parallel to the $p_{y}$ axis.

to this, the photoelectron momentum distributions from helium [Figs. 4(c) and 4(d)] are strongly peaked along the polarization axis of the IR field, reflecting the fact that most of the ionization occurs via electrons that escape over the suppressed Coulomb barrier along the polarization direction.

The WPI that we have observed has a number of similarities and a few important differences as compared to "traditional" WPI. In more conventional WPI, the motion takes place on a purely bound potential surface and the WPI is controlled by changing the delay between pulses. In our case, the delay between attosecond pulses is fixed at one half the IR cycle, but the amplitude and phase of the IR field at which the EWPs are created are easily changed. Also, the EWPs are only transiently bound and so both the total population and the energy-resolved angular distributions can be measured as a function of the various parameters in the experiment and compared to theory. Our scheme offers a unique tool to study the behavior of electrons in a strongly driven atom or molecule, since the EWPs are created in the center of the potential well at a wellcontrolled time.

A number of modifications to the experiments we have presented here are accessible in the near future. For instance, the wavelength of the laser field can be varied, perhaps all the way to the midinfrared, which would allow the time difference between the attosecond pulses to be varied. Most importantly perhaps, it should be possible to study the WPI as a function of the APT duration as was done in the theoretical calculations. This could be done in a polarization gating scheme by varying the gate duration [23].
In conclusion, we have demonstrated that excitation or ionization dynamics can be controlled using an APT in combination with an IR field. Previous attosecond experiments have used the UV pulse to control the time at which an ionization process takes place [4-8,24]. The control demonstrated in this experiment is, to the best of our knowledge, the first use of an attosecond pulse to modulate the probability of an ionization event. When coupled to angular-resolved photoelectron distributions it opens the way for studies of the detailed dynamics of ultra broadband EWPs in driven atomic and molecular systems.

We are grateful to $\mathrm{S}$. Thorin, Dr. F. Lépine, and Professor M. J. J. Vrakking for help with the imaging spectrometer. This research was supported by the Marie Curie Research Training Network (No. MRTNCT-2003-505138, XTRA), the Crafoord Foundation, the Knut and Alice Wallenberg Foundation, the Swedish Research Council and the National Science Foundation (Grant No. PHY-0701372).

*per@eng-johnsson.se

[1] P. M. Paul et al., Science 292, 1689 (2001).

[2] M. Drescher et al., Science 291, 1923 (2001).

[3] M. Uiberacker et al., Nature (London) 446, 627 (2007).

[4] R. Kienberger et al., Science 297, 1144 (2002).

[5] R. Kienberger et al., Nature (London) 427, 817 (2004).

[6] E. Goulielmakis et al., Science 305, 1267 (2004).

[7] P. Johnsson et al., Phys. Rev. Lett. 95, 013001 (2005).

[8] T. Remetter et al., Nature Phys. 2, 323 (2006).

[9] N. F. Scherer et al., J. Chem. Phys. 95, 1487 (1991).

[10] T. Baumert, M. Grosser, R. Thalweiser, and G. Gerber, Phys. Rev. Lett. 67, 3753 (1991).

[11] H. Katsuki et al., Science 311, 1589 (2006).

[12] L. D. Noordam, D. I. Duncan, and T.F. Gallagher, Phys. Rev. A 45, 4734 (1992).

[13] R. R. Jones, C.S. Raman, D. W. Schumacher, and P. H. Bucksbaum, Phys. Rev. Lett. 71, 2575 (1993).

[14] R. López-Martens et al., Phys. Rev. Lett. 94, 033001 (2005).

[15] H. G. Muller, Appl. Phys. B 74, s17 (2002).

[16] A. T. J. B. Eppink and D. H. Parker, Rev. Sci. Instrum. 68, 3477 (1997).

[17] M. J. J. Vrakking, Rev. Sci. Instrum. 72, 4084 (2001).

[18] P. H. Bucksbaum, R. R. Freeman, M. Bashkansky, and T. J. McIlrath, J. Opt. Soc. Am. B 4, 760 (1987).

[19] K. C. Kulander, K. J. Schafer, and J. L. Krause, Atoms in Intense Laser Fields (Academic Press, San Diego, 1992).

[20] J. L. Krause, K. J. Schafer, and K. C. Kulander, Phys. Rev. Lett. 68, 3535 (1992).

[21] K. Kulander and T. Rescigno, Comput. Phys. Commun. 63, 523 (1991).

[22] K. J. Schafer and K. C. Kulander, Phys. Rev. A 42, 5794 (1990).

[23] I. J. Sola et al., Nature Phys. 2, 319 (2006).

[24] M. Drescher et al., Nature (London) 419, 803 (2002). 